Pensamiento Crítico N. ${ }^{\circ}$ 13, pp. 57-70

\title{
Determinantes del proceso de emprendimiento empresarial femenino en el Perú
}

\author{
Juan León Mendoza y David Huari Leasaski
}

\section{RESUMEN}

El presente artículo tiene como objetivo identificar las variables que determinan la probabilidad de que la mujer peruana adulta y jefa de hogar decida emprender algún negocio o empresa propia. Con información de la Encuesta Nacional de Hogares (ENAHO), utilizando la técnica econométrica del modelo logit y el odds ratios, encontramos que la probabilidad de que la mujer emprenda negocios está determinada, en sentido directo, por la edad, el capital social personal, el tamaño de la familia y, en sentido inverso, por el nivel de educación alcanzada y el ingreso per cápita del hogar.

Palabras clave: Emprendimiento, emprendimiento femenino.

\section{ABSTRACT}

This article aims to identify the variables that determine the probability that the adult Peruvian woman head and householder decides to start any business or own enterprise. With information from the National Household Survey (ENAHO), 


\section{Pensamiento Crítico N. 13}

using the econometric technique of logit model and odds ratios, we find that the probability that a woman start a business is determined, in a direct sense, by age, personal social capital, the family size; and, conversely, the level of education attained and household per capita income.

Keywords: Entrepreneurship, female entrepreneurship.

\section{Introducción}

Según el informe mundial del GEM 2008 - Global Entrepreneurship Monitor 2008 Global Report, la población femenina peruana está entre las más emprendedoras del mundo. El 23.6\% de las mujeres entre 18 y 64 años está inmersa en alguna actividad de negocios nacientes o nuevos.

Este hecho permite preguntarse acerca de los factores o variables que condiciona la decisión de las mujeres peruanas a emprender actividades empresariales.

Por ello, el presente artículo tiene como objetivo identificar a las variables que determinan la decisión y capacidad de emprendimiento empresarial de la población femenina peruana.

\section{Percepciones y características generales del emprendimiento empresarial peruano}

El Global Entrepreneurship Monitor, estila medir anualmente el grado de percepción, actitud y de actividad emprendedora de la población adulta masculina y femenina entre 18 a 64 años, para un conjunto de países relevantes en el mundo. Por ejemplo, el último GEM del año 2009 (Bossa y Levie, 2010), incluye 54 países, de las cuales 13 son latinoamericanos.

Según GEM 2009, el 61\% de los peruanos adultos percibe buenas oportunidades para iniciar una empresa o negocio en la zona donde vive. Tal porcentaje, a nivel mundial, representa el tercero más alto y, el primero a nivel de países latinoamericanos (véase Cuadro 1). 


\section{Juan León Mendoza y David Huari Leasaki}

Asimismo, el $74 \%$ de los peruanos adultos cree poseer las habilidades y los conocimientos requeridos para iniciar un negocio; el $32 \%$ percibe la existencia de oportunidades, pero señala que el miedo al fracaso es un disuasivo para formar el negocio; el 32\% de los que no están involucradas en algún negocio tiene intenciones de establecerlos en los próximos tres años; el $88 \%$ está de acuerdo con la afirmación de que, en el Perú, la mayoría de la población considera que comenzar un negocio es una elección de carrera deseable; el $75 \%$ está de acuerdo con la afirmación de que la mayoría de la población considera que los emprendedores gozan de un alto status y reconocimiento social.

Finalmente, el $85 \%$ de los peruanos adultos está de acuerdo con la afirmación de que los medios de comunicación exhiben con frecuencia historias de nuevos negocios exitosos. Dicho porcentaje es el segundo más alto del mundo después de Yemen y el primero a nivel de América Latina.

Cuadro 1. Perú: actitudes y percepciones emprendedoras.

\begin{tabular}{|l|c|c|c|}
\hline \multicolumn{1}{|c|}{ Indicadores } & $\begin{array}{c}\text { Tasa } \\
\mathbf{\%}\end{array}$ & $\begin{array}{c}\text { Lugar en } \\
\text { el ranking } \\
\text { mundial }\end{array}$ & $\begin{array}{c}\text { Lugar en el } \\
\text { ranking América } \\
\text { Latina }\end{array}$ \\
\hline Percepción de oportunidades & 61 & 3 & 1 \\
\hline Percepción de capacidades & 74 & 6 & 3 \\
\hline Miedo al fracaso & 32 & 23 & 3 \\
\hline Intenciones emprendedoras & 32 & 8 & 3 \\
\hline $\begin{array}{l}\text { Emprendimiento como opción de carrera } \\
\text { deseable }\end{array}$ & 88 & 6 & 2 \\
\hline Alto estado de emprendedores exitosos & 75 & 19 & 5 \\
\hline $\begin{array}{l}\text { Atención que los medios de comunicación } \\
\text { le dan el emprendimiento }\end{array}$ & 85 & 2 & 1 \\
\hline
\end{tabular}

Elaboración propia en base al GEM 2009.

Con relación a la actividad emprendedora, el reporte de GEM 2009 indica que el porcentaje de la población peruana entre 18 a 64 años, que está activamente involucrado en crear un negocio, pero que aún no ha pagado sueldos o remuneraciones 


\section{Pensamiento Crítico N. ${ }^{\circ}$ I3}

por más de tres meses, es de $16,1 \%$, el mismo que es la segunda cifra más alta entre los países de América Latina (véase Cuadro 2); en el mismo sentido, el 5,1\% es dueño de un nuevo negocio que ha pagado remuneraciones salariales por un período entre 3 y 42 meses; el 7,5\% es actualmente propietario y gestor de un negocio establecido y que ha pagado remuneraciones salariales por más de 42 meses; en tanto que 7,1\% discontinuó un negocio en los últimos doce meses.

Un indicador relevante que refleja el nivel de la tasa de emprendimiento empresarial y que goza de mucha popularidad es la tasa de actividad emprendedora en etapas iniciales (TEA, por su sigla en inglés). Este indicador mide el porcentaje de la población adulta (18 a 64 años) que es un emprendedor naciente o propietario y gestor de un nuevo negocio. La TEA para Perú es 20,9\%, el mismo que a nivel de los países de América Latina representa la cuarta tasa más alta después de Guatemala, Jamaica y Colombia.

Cuadro 2. Perú: actividad emprendedora.

\begin{tabular}{|l|c|c|c|}
\hline \multicolumn{1}{|c|}{ Indicadores } & $\begin{array}{c}\text { Tasa } \\
\mathbf{\%}\end{array}$ & $\begin{array}{c}\text { Lugar en } \\
\text { el ranking } \\
\text { mundial }\end{array}$ & $\begin{array}{c}\text { Lugar en } \\
\text { el ranking } \\
\text { América Latina }\end{array}$ \\
\hline Actividad emprendedora naciente & 16,1 & 3 & 2 \\
\hline Nuevos negocios & 5,1 & 20 & 10 \\
\hline Actividad emprendedora en etapas iniciales (TEA) & 20,9 & 6 & 4 \\
\hline Negocios, gerentes y propietarios establecidos & 7,5 & 21 & 7 \\
\hline Tasa de discontinuación & 7,1 & 48 & 9 \\
\hline
\end{tabular}

Elaboración propia en base a GEM 2009.

La TEA masculina es ligeramente mayor que la TEA femenina de manera que el éste equivales al 0,86 de la TEA masculina.

\section{Marco teórico y antecedentes}

La palabra emprendedor tiene su origen en el vocablo francés entrepreneur (pionero). Actualmente, el Diccionario de la Academia de la Lengua Española define al 


\section{Juan León Mendoza y David Huari Leasaki}

emprendedor como el "que emprende con resolución acciones dificultosas o azarosas", de manera que emprender consiste en "acometer y comenzar una obra, un negocio, especialmente si encierran dificultad o peligro".

En términos económicos, el emprendedor empresarial podemos definirlo como aquella persona que planea, ejecuta y desarrolla un proyecto de negocios o empresa, con la finalidad de aprovechar oportunidades de mercado o satisfacer necesidades de generación de ingreso propio. De ahí que la metodología GEM especifica la existencia de dos tipos de emprendimiento empresarial: por oportunidad y por necesidad.

El conjunto de variables que determinan la decisión de emprender un negocio, suelen ser clasificadas en dos grupos: factores de entorno y los factores personales correspondientes al emprendedor.

Según la sistematización efectuada por García et. al (2004), los factores de entorno comprenden a: las economías de localización, las economías de urbanización, el entorno financiero, la intervención y la regulación económica, la coyuntura económica, el capital social, la cultura empresarial de la sociedad, la capacidad investigadora/innovadora regional, la estructura sectorial de la actividad económica, los movimientos de población e inmigración, la estructura empresarial por tamaño de las empresas. En tanto que entre los factores personales se incluye a: la necesidad de conseguir logros, el control interno, la tolerancia a la ambigüedad y la capacidad para asumir riesgos, la orientación a la oportunidad, el fuerte compromiso, la insatisfacción con el trabajo asalariado, la seguridad personal, la formación, el entorno familiar y social, la edad, la configuración del equipo directivo, el temor al fracaso y el fracaso empresarial, la capacidad de financiación.

En la línea de los factores de entorno, Lounsbury (1998) señala que los aspectos sociales y culturales condicionan las actitudes de emprendimiento de las personas. Benavente (2008) resume que entre las variables que condicionan la entrada de nuevas empresas al mercado, por ende emprender nuevos negocios, están: la existencia de economías de escala, la concentración de mercado, crecimiento o decrecimiento de mercado, el ciclo económico.

A nivel de las investigaciones de corte empírico, en la línea de los factores personales, Kantis (2004), sobre la base de un estudio efectuado para un grupo de países de América 


\section{Pensamiento Crítico N. 13}

Latina, concluye que entre las principales motivaciones para emprender un negocio están: lograr la realización personal, poner en práctica sus conocimientos, mejorar su nivel de ingresos, contribuir a la sociedad, ser su propio jefe, ser adinerado, ser como un empresario que admiraba, obtener status social, seguir la tradición familiar de estar en los negocios, estar desempleado, no poder estudiar. En el caso peruano, en el marco del GEM, el estudio efectuado por ESAN (2010) encuentra que los principales motivos para el emprendimiento empresarial por oportunidad son: el interés de obtener mayor nivel de ingreso y el deseo de independencia.

Según Blanchflower y Oswald (1998), la falta de capital es una de las variables más importantes que condicionan o limitan el deseo de emprender un negocio. En tanto que la educación -que está relacionada al capital humano- afecta en sentido inverso la probabilidad de convertirse en empresario (Evans y Jovanovic, 1989).

En un estudio efectuado para Colombia, Destré y Henrard (2004), citado por Guataquí et. al (2009) encuentra que la probabilidad emprendedora de generar ingreso propio depende, en sentido inverso, del nivel de la educación y, en sentido directo, de la edad, la tenencia de hijos menores de 16 años y cuando trabaja la pareja del hogar.

\section{El modelo}

Tomando en cuenta lo expuesto en el acápite anterior y, en la línea del enfoque teórico de los recursos y capacidades desarrollado, entre otros, por Barney (1991) para explicar el caso de la capacidad competitiva de las empresas, a continuación formulamos un modelo simple y general que explica la capacidad de emprendimiento empresarial de las personas.

Dada las variables de entorno, se asume que la actitud de emprendimiento empresarial (empren) depende básicamente de un conjunto de factores directamente personales, en especial de su dotación de recursos (activos) o capitales (Ki). La dotación de capital condiciona la capacidad emprendedora y, por ende, su propensión a emprender algún negocio o empresa propia.

$$
\text { empren }=\mathrm{f}(\mathrm{Ki})
$$




\section{Juan León Mendoza y David Huari Leasaki}

El capital puede ser de carácter tangible o intangible. En términos generales, se puede especificar los siguientes tipos de capital: capital humano, capital social, capital financiero, capital físico, capital familiar, capital conyugal, capital institucional, entre otros.

El capital humano comprende al stock de conocimientos y habilidades personales; el capital social es el conjunto de lazos sociales e institucionales de la persona; el capital financiero tiene que ver con el flujo de ingresos acumulados o stock de activos financieros; el capital físico es el conjunto de activos físicos o bienes de capital; el capital familiar comprende al número de miembros del hogar con capacidad de generar ingresos; el capital conyugal se refiere a la tenencia de una pareja estable (en calidad de casados o de convivencia) que permite generar una especie de asociación o alianza estratégica de pareja; y el capital institucional está relacionada con el conjunto de reglas, normas o conductas personales.

En el marco del modelo general formulado, se especifica un modelo en el que la decisión de emprendimiento empresarial (empren) depende de la dotación del capital humano (Kh), el capital social (Ks), el capital familiar (Kf), el capital de la riqueza financiera $(\mathrm{Kr})$ y el capital conyugal $(\mathrm{Kc})$ :

$$
\text { empren }=\mathrm{f}(\mathrm{Kh}, \mathrm{Ks}, \mathrm{Kf}, \mathrm{Kr}, \mathrm{Kc})
$$

\section{Aspectos metodológicos}

Con la finalidad de identificar y cuantificar la importancia explicativa de las variables personales o familiares que condicionan la decisión de emprendimiento empresarial, se efectúa una regresión econométrica utilizando el modelo logit.

Los datos a considerarse en la regresión provienen de la ENCUESTA NACIONAL DE HOGARES SOBRE CONDICIONES DE VIDA Y POBREZA (ENAHO, 2008). Esta encuesta se ejecuta en los 24 departamentos del Perú, tanto en el área Urbana como en el Rural y tiene como población objetivo al conjunto de viviendas y sus ocupantes, excluyéndose a los miembros de las fuerzas armadas, entre otros.

La base de dato final contiene principalmente los módulos de empleo (modulo 500 ) y de participación ciudadana (modulo 800a). La base del 2008 cuenta con un total 


\section{Pensamiento Crítico N. 13}

de 63 mil observaciones aproximadamente de los cuales 3 mil 547 corresponden a la muestra del estudio, vale decir, las mujeres ocupadas jefes de hogar.

La ecuación a regresionar es aquella que resulta de especificar el modelo teórico formulado, por lo que se plantea el siguiente modelo econométrico:

$$
\begin{aligned}
\text { empren }= & \beta 0+\beta_{1}(\text { edad })+\beta_{2}\left(\text { edad }^{2}\right)+\beta_{3}\left(\text { Ieduca }^{2}\right)+\beta_{4}\left(\text { Ieduca }^{3}\right)+\beta_{5}\left(\text { (leduca }^{4}\right) \\
& +\beta_{6}(\text { vínculo })+\beta_{7}(\text { tam })+\beta_{8}(\text { Linghog })+\beta_{9} \text { participa }+u
\end{aligned}
$$

empren (emprendimiento empresarial). Esta es una variable binaria que toma el valor de uno si la mujer jefa de hogar es emprendedora (trabaja como empleadoras o trabajadores independientes con personas a su cargo) y de cero en caso contrario. La data se genera a partir de la pregunta p507, p508, p509 y p510 del ENAHO 2008.

edad y edad ${ }^{2}$. La edad se considera como una variable proxi de la experiencia laboral. La experiencia laboral condiciona la acumulación de capital humano. También se considera la edad al cuadrado (edad2), para ver si el aporte de esta variable de capital humano sobre la probabilidad de ser emprendedora es creciente o decreciente.

Ieduca. Es la variable educación, que también se considera como un elemento que está relacionada a la acumulación de capital humano. Esta variable es policotoma y puede tomar cuatro valores: uno para las personas sin nivel educativo, dos para el nivel primario, tres para el secundario y cuatro para el nivel superior. En este último caso se incluye el nivel superior universitario y no universitario. En cada nivel se toma en cuenta los estudios culminados. En la regresión se consideran a los estudios primarios (Ieduca2), secundarios (Ieduca3) y superiores (Ieduca4) alcanzadas por la mujer.

vínculo. Representa al capital conyugal. Es una variable dicotómica que toma el valor de uno si la mujer está casada o es conviviente y de cero en otros casos.

tam. Es el tamaño de la familia y se considera como una variable del capital familiar. Se determina mediante el número de miembros en el hogar. 


\section{Juan León Mendoza y David Huari Leasaki}

linghog. Logaritmo del ingreso per cápita del hogar. Este es una variable proxi del capital financiero y se construye como un cociente del ingreso total por hogar entre el número de miembros en el mismo. Se considera en términos de logaritmos a fin de alisar la serie y cambiar la escala de los valores.

participa. Participación de la mujer en alguna organización social o institucional. Ésta es una variable que representa al capital social de la persona. Toma el valor de uno si participa en alguna organización y de cero en caso contrario.

\section{Determinantes del emprendimiento empresarial femenino: resultado econo- métrico}

En los casos en que para una parte de la muestra no existen observaciones en la variable dependiente, en el proceso de estimación econométrica de los parámetros, se utilizan los modelos logit o probit (Gujarati, 2004). Ambos son buenos sustitutos en cuanto a la modelación discreta y los resultados que arrojan son similares.

Sin embargo, efectuada las regresiones con ambos modelos, se encontró que el mejor modelo para este estudio es el logit, debido a que los estadísticos AIC y BIC del modelo logit son 4310.822 y 4372.50 respectivamente, lo cual es menor que 4314.441 y 4376.180 en el caso del modelo probit.

El resultado de la regresión se muestra en el Cuadro 3. En general los coeficientes resultan estadísticamente significativos, a excepción de la variable vínculo. Según el estadístico $\mathrm{Chi} \wedge 2$, las variables incluidas en este modelo son conjuntamente significativas para explicar la probabilidad de que la mujer sea emprendedora. Además, el test de Wald y el de verosimilitud (LR) advierten evidencia estadística significativa de que el modelo es adecuado. 


\section{Pensamiento Crítico N. ${ }^{\circ}$ I3}

Cuadro 3. Determinantes del emprendimiento femenino: modelo Logit.

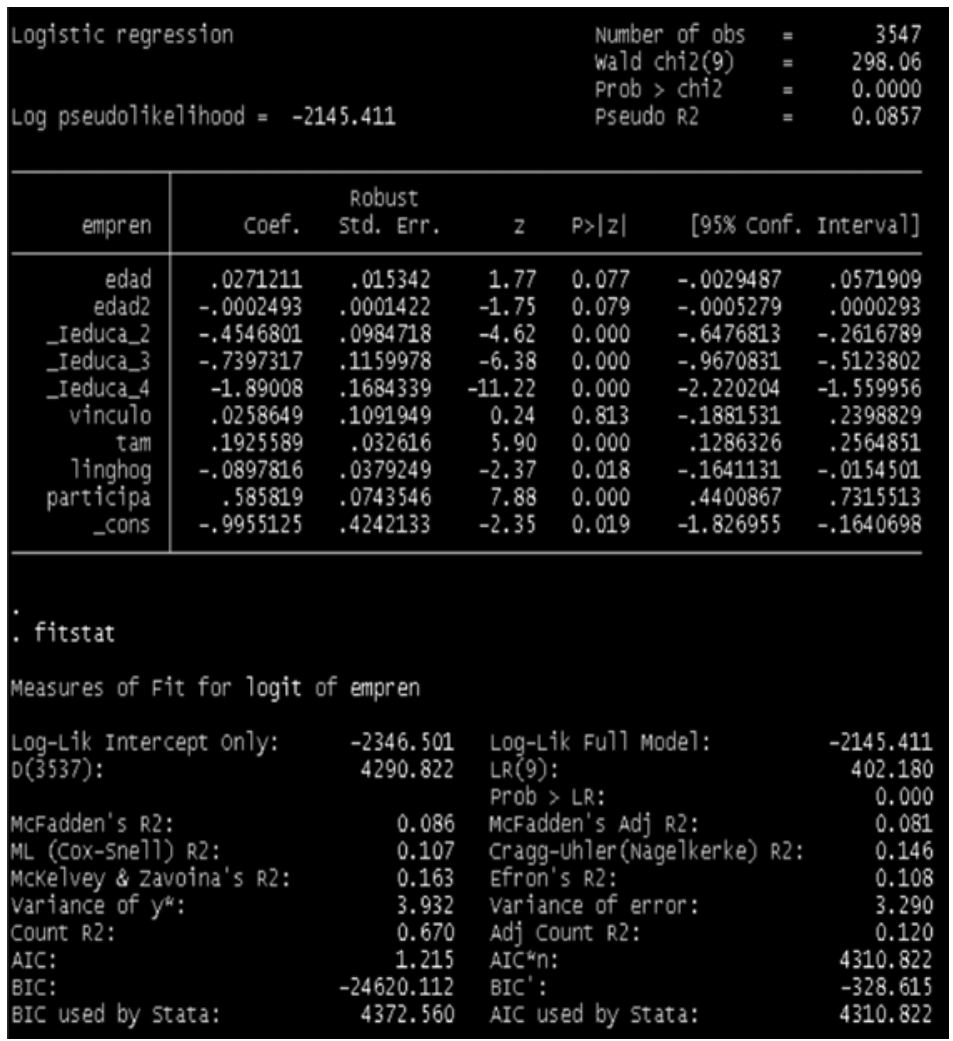

Con la finalidad de evaluar el grado de precisión de los resultados hallados, aplicamos el test de significancia correspondiente a la variable participa. El test de Wald arroja un $\mathrm{Chi}^{\wedge} 2=62,17$ y el de Verosimilitud un $\mathrm{Chi} \wedge 2=62,74$. En ambos casos la probabilidad estimada del estadístico es menor que 0,05, por lo cual se rechaza la hipótesis nula de que el efecto de la variable participa sobre la decisión emprendedora no es estadísticamente significativa. Asimismo, dado que la prueba de Verosimilitud es más potente que el test de Wald, dicha prueba nos permite concluir que el modelo sin restricción presenta mayor verosimilitud, de manera que también se maximiza la probabilidad de que la obtención de los datos sea más exacta. 


\section{Juan León Mendoza y David Huari Leasaki}

Los parámetros estimados para la variable edad son estadísticamente significativos con un $90 \%$ de confianza. El signo positivo para dicha variable nos indica que, a mayor edad, la mujer tiende a decidir emprender algún negocio. Sin embargo, el hecho de que el parámetro de edad al cuadrado sea de signo negativo, indica que dicho grado de emprendimiento se incrementa, pero, a una tasa decreciente.

La regresión muestra que la educación alcanzada por la mujer influye de manera negativa sobre la decisión de emprendimiento empresarial, en forma similar que el ingreso per cápita del hogar.

El tamaño del hogar (número de miembros de la familia) y la pertenencia a alguna organización social influyen en sentido directo sobre la decisión de emprender algún negocio.

En los modelos no lineales de respuesta binaria, los parámetros estimados tienen poca utilidad para efectos de análisis, debido a que no cuantifican directamente el incremento en la probabilidad dado un cambio marginal en una variable explicativa; por ello es necesario el cálculo de las derivadas parciales o los efectos marginales. Los efectos marginales o elasticidades suelen proporcionar una buena aproximación del cambio registrado en la variable endógena respecto de un cambio en alguna de las exógenas.

Sin embargo, en el marco en que los coeficientes estimados por el modelo logit no son iguales a la magnitud de las variaciones en la probabilidad, se pueden estimar directamente las elasticidades con la metodología odds ratios. Este indicador se obtiene a través de la linealización del modelo y se define como el cociente de las probabilidades de que se elija la opción uno, frente a la posibilidad de que se elija la opción cero.

Las odds ratios nos permite medir la probabilidad de que ocurra un suceso frente a la probabilidad de que no ocurra, condicionadas ambas probabilidades a un suceso determinado. En el caso de nuestro estudio, permite medir la probabilidad de que una mujer decida emprender algún negocio frente a la probabilidad de no hacerlo condicionada por sus dotaciones de capital o activos personales. Las estimaciones hechas se muestran en el Cuadro 4. 


\section{Pensamiento Crítico N. I3}

Cuadro 4. Determinantes de la probabilidad de emprendimiento empresarial femenino: método odds ratios.

\begin{tabular}{|c|c|c|c|c|c|c|}
\hline empren & b & $z$ & $P>|z|$ & $\%$ & Xstdx & SDofX \\
\hline $\begin{array}{r}\text { edad } \\
\text { edad2 } \\
\text { _Ieduca_2 } \\
\text { _Ieduca_3 } \\
\text { _Ieduca_4 } \\
\text { vinculo } \\
\text { tam } \\
\text { 1inghog } \\
\text { participa }\end{array}$ & $\begin{array}{r}0.02712 \\
-0.00025 \\
-0.45468 \\
-0.73973 \\
-1.89008 \\
0.02586 \\
0.19256 \\
-0.08978 \\
0.58582\end{array}$ & $\begin{array}{r}1.768 \\
-1.754 \\
-4.617 \\
-6.377 \\
-11.221 \\
0.237 \\
5.904 \\
-2.367 \\
7.879\end{array}$ & $\begin{array}{l}0.077 \\
0.079 \\
0.000 \\
0.000 \\
0.000 \\
0.813 \\
0.000 \\
0.018 \\
0.000\end{array}$ & $\begin{array}{r}2.7 \\
-0.0 \\
-36.5 \\
-52.3 \\
-84.9 \\
2.6 \\
21.2 \\
-8.6 \\
79.6\end{array}$ & $\begin{array}{r}49.7 \\
-32.9 \\
-17.4 \\
-25.0 \\
-47.9 \\
0.9 \\
31.1 \\
-11.4 \\
34.0\end{array}$ & $\begin{array}{r}14.8868 \\
1597.8778 \\
0.4211 \\
0.3892 \\
0.3448 \\
0.3617 \\
1.4077 \\
1.3449 \\
0.5001\end{array}$ \\
\hline
\end{tabular}

Según los resultados de la estimación, un año adicional en la edad de la mujer, manteniendo constante las demás variables, aumenta en $2,7 \%$ la probabilidad de que ésta sea emprendedora frente a la probabilidad de no serlo. El porcentaje negativo en la edad al cuadrado nos indica que cada año adicional de la mujer influye positivamente sobre la probabilidad de emprender, pero, el aporte es cada vez menor.

Respecto a la variable educación, los resultados indican que si la mujer alcanza los niveles de educación primaria, secundaria o superior, su probabilidad de emprender un negocio disminuye en $36,5 \%, 52,3 \%$ y $84,9 \%$ respectivamente. Cuanto mayor es el nivel educación formal alcanzada por la mujer, es menor la probabilidad de que emprenda algún negocio.

El que a mayor educación se tenga menor probabilidad de emprendimiento empresarial, estaría reflejando el hecho de que una mujer con mayor nivel de educación tiene mayores oportunidades laborales o de encontrar empleo adecuado, de manera que su motivación de emprender un negocio o crear una empresa por necesidad tiende a disminuir. 


\section{Juan León Mendoza y David Huari Leasaki}

En el mismo sentido, el que la probabilidad de emprender un negocio sea de $-8,6 \%$ en la medida en que aumenta el nivel del ingreso per cápita en el hogar, estaría reflejando también el hecho de que una mujer que pertenece a un hogar de mayor nivel de ingreso, tiene menor necesidad de crear algún negocio propio.

Con relación a la variable tam, el coeficiente estimado indica que por cada miembro adicional en el hogar de la mujer adulta en la que ella es la jefa, su probabilidad de emprender un negocio propio aumenta en $21,2 \%$.

Finalmente, las mujeres que pertenecen o participan en alguna organización y/o asociación, tienen un $79,6 \%$ más de probabilidad de emprender un negocio que aquellas que no cuentan con dicho capital social.

En resumen, la probabilidad de que una mujer adulta y jefa de hogar emprenda algún negocio es mayor en la medida en que tenga una mayor edad, aumente el número de miembros en el hogar y participe en alguna organización social. En tanto que disminuirá, dicha probabilidad, cuando alcance mayores niveles de educación e ingreso familiar per cápita.

\section{Bibliografía}

Barney, Jay (1991). "Firm Resources and Sustained Competitive Advantage", Journal of Management, vol. 17, N. ${ }^{\circ}$ 1, pp. 99-120.

Benavente, José (2008). La dinámica empresarial en Chile 1999-2006. Ministerio de Economía, Gobierno de Chile, Santiago.

Blanchflower, D., y A. Oswald (1998). "What makes an entrepreneur?" Journal of Labor Economics, 16:1, pp. 26-60.009 Ernesto Amorós / Carla Bustamante / Germán Echecopar / Daniela Ortega

Bossa. N., y J. Levie (2010). Global entrepreneurship monitor, 2009 Global Report. Babson Collegue, Universidad del Desarrollo, Raykjavik University. Disponible en: http://www.gemconsortium.org/download/1287158832171/GEM\%202009\%20 Global\%20Report\%20Rev\%20140410.pdf 


\section{Pensamiento Crítico N. 13}

ESAN (2010). Global entrepreneurship monitor Perú 2008, Centro de Desarrollo Emprendedor, Lima.

Evans, D. y B. Jovanovic (1989). "An estimated model of entepreunerial choice under liquidity constrains." Journal of Political Economy, 79:4, pp. 807-27.

García, C., A. Martínez, y R. Fernández (2004). Análisis de los factores determinantes de la creación de empresas: una evidencia empírica en Castilla y León. Disponible en: http://www.google.com.pe.

Guataquí, J., A. García, M. Rodriguez (2009). Estimaciones de los determinantes de los ingresos laborales en Colombia con consideraciones diferenciales para asalariados y cuenta propia. Serie Documento de Trabajo N. ${ }^{\circ}$ 70, Universidad del Rosario, Colombia. Disponible: http://www.banrep.gov.co/documentos/conferencias/2009/ guataqui.pdf

Gujarati, Damodar (2003). Econometría. McGraw Hill, México.

Kantis, Hugo (2004). Desarrollo emprendedor: América Latina y la experiencia Internacional. Nomos, Bogotá.

Lounsbury, Michael (1998). "Collective entrepreneurship: the mobilization of college and university recycling coordinators". Journal of Organizational Change Management, 11(1): 50-69. 